\title{
Surface plasmon optoelectronics and exceptional point waveguides on silicon
}

Pierre Berini

Pierre Berini, "Surface plasmon optoelectronics and exceptional point waveguides on silicon," Proc. SPIE 11031, Integrated Optics: Design, Devices, Systems, and Applications V, 1103107 (26 April 2019); doi: 10.1117/12.2524502

SPIE. Event: SPIE Optics + Optoelectronics, 2019, Prague, Czech Republic 


\title{
Surface plasmon optoelectronics and exceptional point waveguides on silicon

\author{
Pierre Berini \\ School of Electrical Engineering and Comp. Sci., University of Ottawa, Ottawa, K1N 6N5, Canada \\ Department of Physics, University of Ottawa, Ottawa, K1N 6N5, Canada \\ Center for Research in Photonics, University of Ottawa, Ottawa, K1N 5N6, Canada \\ berini@eecs.uottawa.ca
}

\begin{abstract}
We discuss recent progress on plasmonic and metasurface optoelectronic devices on silicon. Emphasis is placed on devices for sub-bandgap hot-carrier photodetection and high-speed intensity modulation. Surface-normal structures are of particular interest. We also discuss recent work on exceptional point waveguides, wherein the modal evolution traces out adiabatic and anti-adiabatic parametric paths around an exceptional point, and in combination with a gain saturation nonlinearity, leads to non-reciprocity.
\end{abstract}

Keywords: Surface plasmons, optoelectronics, metasurfaces, semiconductor, metal, exceptional point

\section{INTRODUCTION}

Metallic nanostructures such as antennas [1,2] and gratings [3-5] can be designed to operate effectively as a coupling structure for incident beams to surface plasmon polaritons (SPPs) propagating or resonating thereon. On a semiconductor, such nanostructures can act simultaneously as a device electrode while ensuring strong optical field overlap with the active region. Moreover, SPP fields can be confined to sub-wavelength regions and enhanced relative to the exciting field.

These features are very attractive for application to optoelectronic devices such as in photodetectors [6-14] and modulators [15-18] because the involvement of SPPs alters conventional trade-offs between responsivity and speed, or modulation and speed, respectively. Fundamentally, sub-wavelength confinement enables the active region to be shrunk to nano-scale dimensions, while the SPP field enhancement can maintain good optoelectronic performance.

Here we discuss progress on surface plasmon enhanced photodetectors and modulators, particularly, recent proposals for a photodetectors exhibiting THz electrical bandwidths [8,9], demonstrations of sub-bandgap photodetectors on Si [12$14]$, and high-speed reflection modulators on Si based on the carrier refraction effect [16,17].

\section{PHOTODETECTORS}

A Schottky photodiode on n-doped semiconductor is sketched in Fig. 1(a). Two detection mechanisms are frequently exploited for photodetection [6]. The first is photodetection by the creation of electron-hole pairs (EHPs) in the semiconductor due to the absorption of incident photons of energy greater than the bandgap energy of the semiconductor $\left(h v>E_{g}\right)$. This mechanism involves the creation of EHPs, the separation of the EHPs by a bias field, and carrier drift/diffusion in the semiconductor. The second mechanism is photodetection by the internal photoemission (IPE) of energetic carriers excited in the metal by absorption of incident quanta. The energy band diagram sketched in Fig. 1(b), for a contact to $\mathrm{n}$-doped semiconductor, further describes this 3-step process, which is comprised of the photo-excitation of carriers in the metal, transport of excited (hot) carriers with scattering toward the metal interface(s), and emission of hot carriers over the Schottky barrier into the semiconductor. IPE occurs for photon energies greater than the Schottky barrier energy $\Phi_{B}$ and is useful to extend photodetection to photon energies below the semiconductor bandgap energy (i.e., for $\Phi_{B}<h v<E_{g}$ ). 

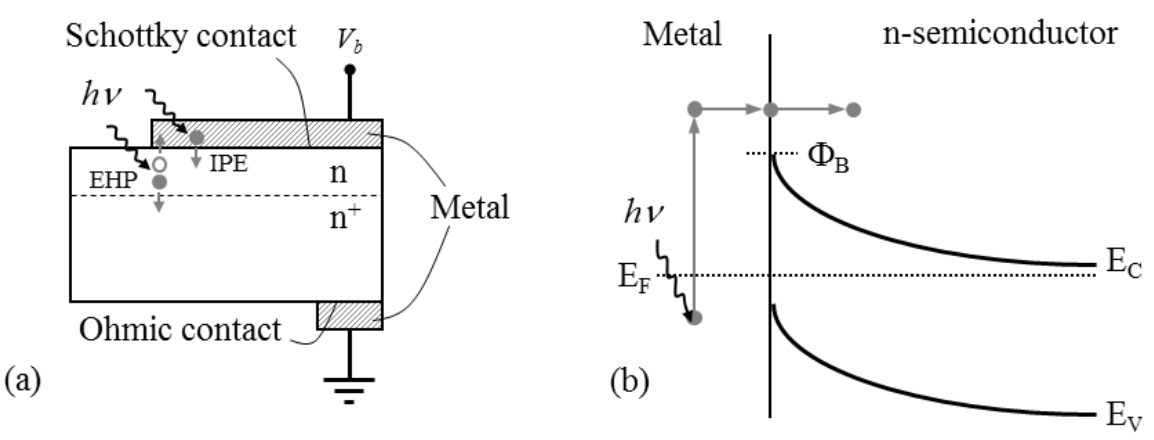

Figure 1. (a) Schottky contact on an n-doped semiconductor illuminated by a beam of photon energy $h v$. Electrons are shown as filled circles and holes by unfilled ones. (b) Energy band diagram of Schottky contact. The 3-step IPE process consists of photo-excitation of carriers in the metal, carrier transport through the metal, and carrier emission into the semiconductor (hot electrons are sketched here). $E_{C}$ and $E_{V}$ are the conduction and valence band edges, $E_{F}$ is the Fermi level and $\Phi_{B}$ is the Schottky barrier height.

\subsection{Photodetectors on III-V}

We propose SPP photodetectors capable of THz electrical bandwidths and high responsivity [8,9]. The photodetectors are based on the enhancement of the electric field in low-defect, low-doped $\operatorname{In}_{0.53} \mathrm{Ga}_{0.47}$ As detection volumes located in the gaps of metal dipole nanoantennas [8], or below metal monopole nanoantennas [9]. In both cases the detection medium (InGaAs) is formed on a non-absorbing InP substrate. In the first case [8], the arms of the dipoles in the array form Schottky contacts to the InGaAs detection volumes, and are connected to independent contacts such that a punchthrough bias field can be applied to the detection volumes [8]. In the second case [9], the monopole nanoantennas are similarly interconnected. Photodetection in both device concepts occurs via the creation of EHPs in the InGaAs volumes.

A maximum responsivity of $\sim 100 \mathrm{~mA} / \mathrm{W}$ at wavelengths near $1550 \mathrm{~nm}$ is predicted [8]. The $3 \mathrm{~dB}$ electrical bandwidth of the device is estimated based on its RC rise time and the hole transit time (slowest carrier) through the detection volume. Given the nano-scale length of the InGaAs detection volumes, and the possibility for ballistic transport therein, the expected electrical bandwidth ranges from $\sim 0.7$ to $4 \mathrm{THz}$. Trends relating the responsivity to the antenna dimensions are observed, highlighting a trade-off between the field-enhancement in the gap and the volume of the detection region.

\subsection{Photodetectors on $\mathrm{Si}$}

We propose a sub-bandgap photodetector consisting of a $\mathrm{Au}$ grating on a thin $\mathrm{Au}$ film on $\mathrm{p}$-Si, which exploits the enhancement associated with the excitation of SPPs at the Au/p-Si interface [13]. The grating is defined via e-beam lithography and Au lift-off, on a Au film formed beforehand by optical lithography, on p-Si. In this device, SPPs are absorbed by the Au film, leading to the creation of hot holes therein that can cross into the p-Si where they are collected as the photocurrent. Results are promising in terms of responsivity, with a value of $13 \mathrm{~mA} / \mathrm{W}$ measured at $1550 \mathrm{~nm}$ - this is among the highest values reported to date for sub-bandgap detectors based on IPE [13]. This detector can be used in, e.g., non-contact wafer probing or in short-reach optical communications applications.

We also propose and investigate a Schottky contact detector where the contact metal is a rectangular metal nanowire supporting SPPs with strong localization at the Schottky contact [12]. We consider p-Si for the semiconductor, Au for the nanowire, operation in the infrared $\left(\lambda_{0} \sim 1550 \mathrm{~nm}\right)$, and IPE of hot holes as the sub-bandgap detection mechanism. We find that the main SPP mode propagating along the Au nanowire exhibits mode fields that are tightly confined to the immediate vicinity of the stripe, to within a very small area. End-fire coupling efficiencies, responsivities, dark currents, and minimum detectable powers are computed [12]. We find that the structure operates as a nanoscale "point detector" and that it may be useful for optical beam scanning applications, motivated by the very small area of the photodetector nanowire mode relative to, e.g., a Gaussian beam. The nanowire field distribution approximates a Dirac delta function in comparison, and the photocurrent originates from selective absorption of the nanowire SPP mode. The proposed application was verified through numerical simulation, demonstrating that a nanoscale "point detector" can scan a tightly focused Gaussian beam $\left(2.5 \mu \mathrm{m}\right.$ diameter at $\left.\lambda_{0} \sim 1550 \mathrm{~nm}\right)$ with good resolution and signal-to-noise [12]. 
Figure 2 shows a schematic of another structure of interest [14]. It consists of an array of monopoles (thickness $t$ ) for confining SPPs on a lightly-doped p-Si epitaxial layer on a heavily-doped $\mathrm{p}^{+-} \mathrm{Si}$ substrate. The Au monopoles are interconnected via Au lines running perpendicular to their axes through the middle of each to a common circular contact pad used to collect the photocurrent. The pad is formed of Au/Ti because Ti has a larger Schottky barrier to p-Si thereby reducing the dark current contributed by this portion of the device. The monopoles are designed to couple normally-incident $p$-polarised infrared ( $\lambda \sim 1550 \mathrm{~nm}$, sub-bandgap) light to SPPs propagating along their bottom surface such that the SPP fields are coincident with the Schottky contact. As SPPs propagate they are absorbed in the metal, generating hot carriers therein primarily along the Schottky contact. Detection then occurs through the collection of hot holes in p-Si via IPE. The substrate is placed on a backside $\mathrm{Al} \mathrm{Ohmic}$ ground contact.

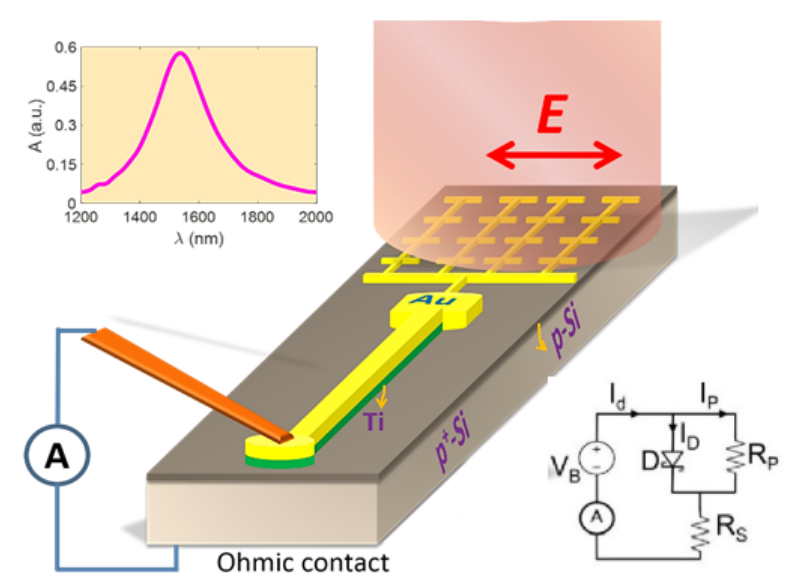

Figure 2. Schematic of an Au/p-Si optical antenna Schottky contact diode for sub-bandgap detection. The materials used are $\mathrm{Au}$ for the antennas, $\mathrm{p}-\mathrm{Si}$ for the epitaxial layer, $\mathrm{p}^{+-} \mathrm{Si}$ for the substrate, Au/Ti for the probing structure (pad and arm), and $\mathrm{Al}$ for the backside Ohmic contact. The device is illuminated from the top with $p$ polarised IR light aligned along monopole lengths. A good design of length $125 \mathrm{~nm}$, width $44 \mathrm{~nm}$ and thickness $20 \mathrm{~nm}$ produces a resonant wavelength at $\lambda=1534 \mathrm{~nm}$ as identified from the computed absorptance response in inset.

The structures were fabricated using a combination of photolithography and e-beam lithography, the former to define the large $\mathrm{Au} / \mathrm{Ti}$ probing features, and the latter to define the Au nanoantennas and interconnect lines. Figs. 3(a) - 3(d) show scanning electron microscope (SEM) images of fabricated structures [14]. The metal thickness and roughness were deduced from atomic force microscope (AFM) scans of the antenna array, as shown in Fig. 5(e), revealing a thickness of $\sim 17 \mathrm{~nm}$ with a root mean square (RMS) roughness of $1 \mathrm{~nm}$ for each monopole (design target $=20 \mathrm{~nm}$ ). All other dimensions are well in keeping with the target ones.

A photodetector under test was biased by probing its probe pad and grounding its $\mathrm{Al}$ contact using a source meter (Fig. 2). A cleaved polarisation-maintaining single-mode fibre (PM-SMF) was used to excite the photodetector under test from the top. The fibre was manipulated using a six-axis micro-positioner. A tunable external cavity laser was used to generate the incident light at $\lambda \sim 1550 \mathrm{~nm}$. The laser was thermally stabilized and linearly polarized, with the polarisation aligned along the slow axis of the PM-SMF. The setup was placed on a vibration isolation optical table and shielded from background light. Instrument control and data acquisition were performed via computer using LabVIEW (SP1 2013)]. The optical power incident on a photodetector was determined by removing the losses of all the elements in the input path of the setup.

The $p$ polarized photocurrent response was measured at normal incidence with $P_{\mathrm{mc}}=1.6 \mathrm{~mW}$ and $V_{\mathrm{B}}=-10 \mathrm{mV}$ for two detectors having arrays of monopoles of different dimensions. Each monopole in both arrays were $\sim 45 \mathrm{~nm}$ wide and $17 \mathrm{~nm}$ thick, but the shorter ones were $\sim 124 \mathrm{~nm}$ long and the longer ones were $130 \mathrm{~nm}$ long. Two wavelength dependencies were observed on each response [14]. A rapidly varying dependency come from Fabry- 
Perot resonances as light transmitted through the array propagates through the semi-transparent substrate $\left(\mathrm{p}^{+-}\right.$ $\mathrm{Si}$ ) and is reflected from the backside Ohmic contact. The second wavelength dependency was a slow one produced by the monopole resonance, where it was observed that increasing the monopole length, red-shifts the resonance wavelength, as expected [14].

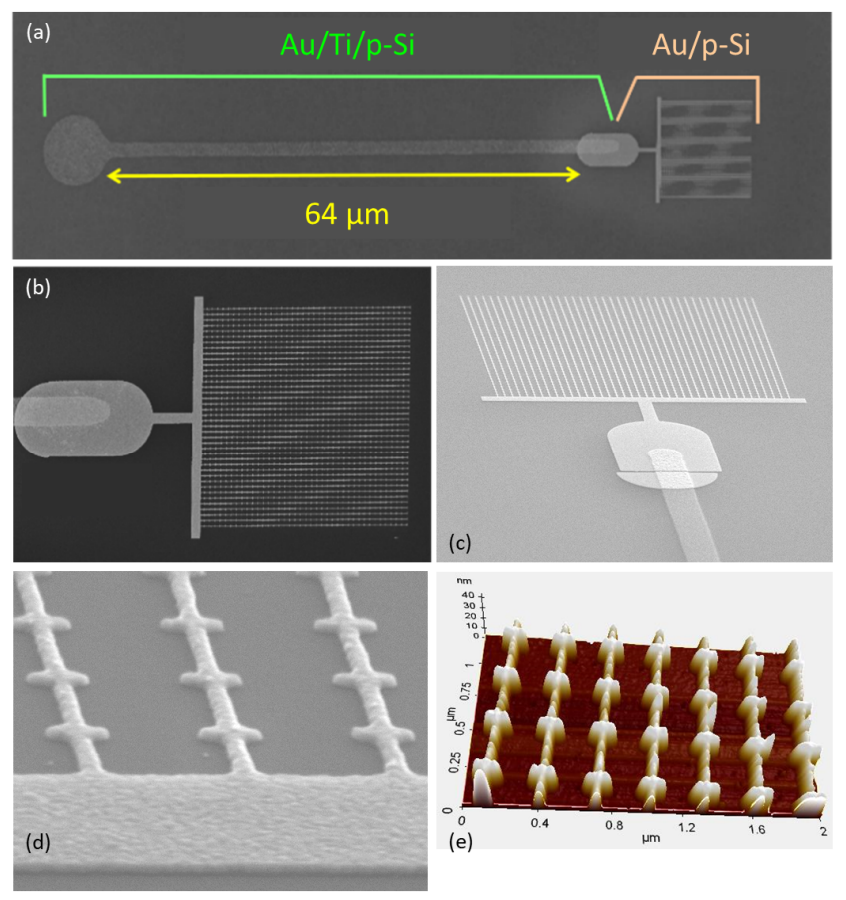

Figure 3. (a)-(d) Scanning electron microscope (SEM) images of fabricated structures. (a) View of a full structure comprising a bilayer Au/Ti probing structure and an array of Au electrically-contacted monopole antennas. (b) and (c) Higher-magnification images of arrays. (d) Higher-magnification image of electrically-contacted monopole antennas. (e) Atomic force microscope image of electrically-contacted monopole antennas.

\section{MODULATORS}

We propose a plasmonic surface that produces an electrically-controlled reflectance as a high-speed optical intensity reflection modulator [16,17]. The device is conceived as a metal-oxide-semiconductor capacitor on silicon with its metal nanostructured as a thin patch bearing a contiguous nanoscale grating. The metal structure serves multiple functions: as a driving electrode, as a grating coupler for perpendicularly-incident $p$-polarised light to surface plasmons, and as a waveguide for supporting SPPs on the underside of the patch.

Modulation is produced by charging and discharging the capacitor and exploiting the carrier refraction effect in Si along with the high sensitivity of strongly confined SPPs to refractive index perturbations in Si. The area of the modulator is set by the area of the incident beam, leading to a very compact device for a strongly-focused beam $(\sim 2.5 \mu \mathrm{m} \mathrm{dia}$.).

The modulator can operate over a broad electrical bandwidth with a modulation depth of 3 to $6 \%$, a loss of 3 to $4 \mathrm{~dB}$, and an optical bandwidth of $\sim 50 \mathrm{~nm}$. About 1000 modulators can be integrated and excited independently over a $50 \mathrm{~mm}^{2}$ area producing an aggregate electro-optic modulation rate in excess of $1 \mathrm{~Tb} / \mathrm{s}$ - Fig. 4(a). We demonstrate modulators operating at telecommunications wavelengths, fabricated as nanostructured $\mathrm{Au} / \mathrm{HfO}_{2} / \mathrm{p}$-Si capacitors - Fig. 4(b) [16,17].

Applications such as short-reach data communications, massively-parallel data output for optical interconnects to chips, and functional wafer probing for compliance testing post-manufacturing are envisaged. Packaging is simplified because butt-coupling and propagation through end-facets are not required, and simpler packages suitable for surface devices can be used. The modulators break conceptually from waveguide-based devices, belonging to the same class of devices as surface photodetectors and vertical cavity surface-emitting lasers. 

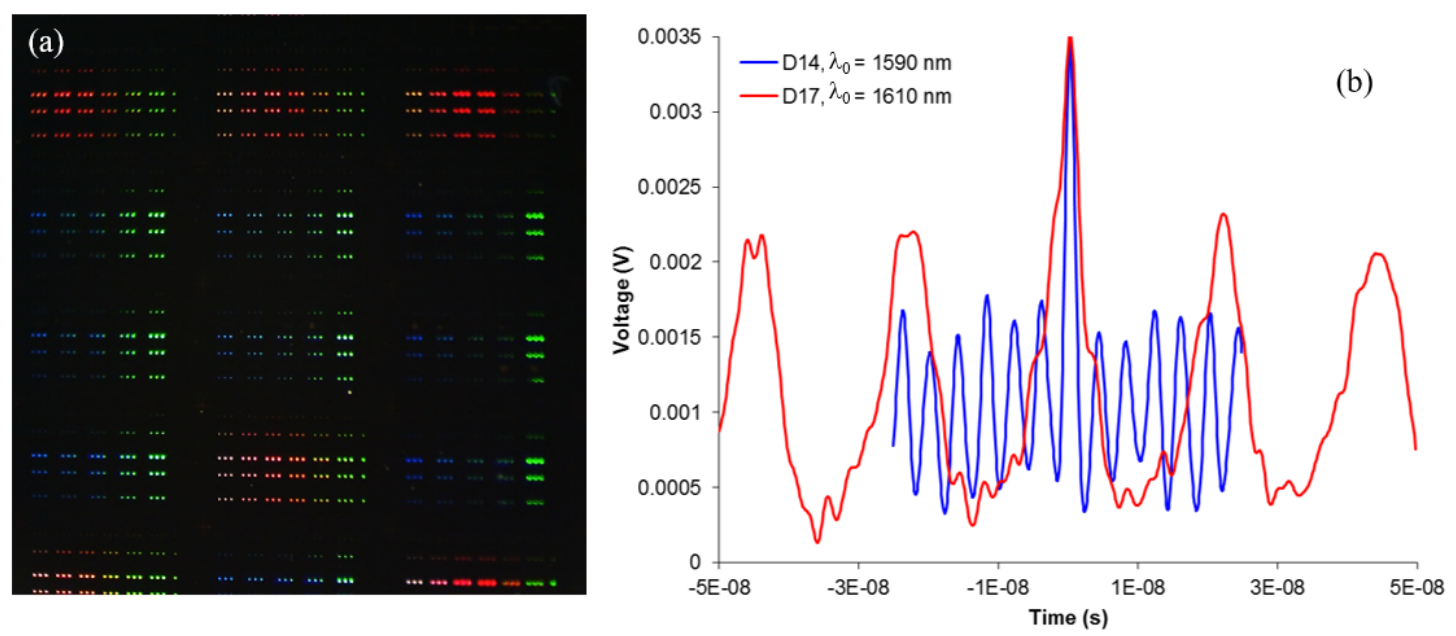

Figure 4. (a) Optical microscope image of modulators of varying grating period. Each colour spot corresponds to one modulator. The area shown is about $100 \mathrm{~mm}^{2}$ and contains $\sim 1700$ modulators. (b) Optical modulation traces measured with an oscilloscope for $\mathrm{Au} / \mathrm{HfO}_{2} / \mathrm{p}$-Si MOS modulators driven at about $1 \mathrm{~V}$.

\section{EXCEPTIONAL POINT WAVEGUIDES}

Synthetic optical materials having a carefully-structured complex refractive index distribution, following non-Hermitian Hamiltonian analogs, have attracted significant attention because of their interesting optical behaviour [19]. Such materials enable functions such as uni-directionality and unconventional beam dynamics.

The interplay between gain and loss, especially in low-loss (long-range) plasmonic systems is interesting because paritytime (PT) symmetric and exceptional point systems become readily accessible, leading to practical experimental demonstrations. Plasmonic waveguide Bragg gratings incorporating structured and unstructured gain media, enabling distributed feedback lasing [20] and single-sided reflection [21] are of particular interest, as are single-sided diffraction gratings implemented as a holographic lattice that transitions through the exceptional point [22].

Interactions based on encircling-an-exceptional-point, which are reciprocal in the linear regime, may become nonreciprocal in the nonlinear regime over a very broad optical bandwidth (limited mainly by the bandwidth of the nonlinearity) [23]. Recent experiments in passive Si-based optical waveguides confirm modal evolution that trace out adiabatic and anti-adiabatic parametric paths around an exceptional point [24].

\section{CONCLUDING REMARKS}

Metal nanoantenna arrays and gratings are effective as metasurfaces for coupling broadside incident beams to SPPs. Such surfaces allow the excitation of SPPs with strongly enhanced fields in very small sub-wavelength active volumes and in combination with a semiconductor active region, lead to compelling devices and applications. Field enhancement allows good optoelectronic performance to be achieved in a small active volume which then leads to fast photodetectors and modulators. These points were made and discussed, and several metasurface optoelectronic devices presented: A modulator based on a silicon MOS structure incorporating a metal grating on a metal film; and photodetectors based on metal dipole and monopole arrays on III-V, on a grating on a metal film on Si, and on a nanowire on Si. In all cases, the metallic nanostructures were also used as device electrodes, either to collect photocurrent current or drive the device. We also discussed recent work on exceptional point waveguides, wherein the modal evolution traces out adiabatic and antiadiabatic parametric paths around an exceptional point, and in combination with a gain saturation nonlinearity, leads to non-reciprocity.

\section{REFERENCES}

[1] P. Bharadwaj, B. Deutsch and L. Novotny, “Optical antennas,” Adv. Opt. Photon. 1, 438-483 (2009). 
[2] S. Siadat Mousavi, P. Berini, and D. McNamara, "Periodic plasmonic nanoantennas in a piecewise homogeneous background," Optics Express 20, 18044-18065 (2012).

[3] G. Lévêque, and O. J. F. Martin, "Optimization of finite diffraction gratings for the excitation of surface plasmons," J. Appl. Phys. 100, 124301 (2006).

[4] I. P. Radko, S. I. Bozhevolnyi, G. Brucoli, L. Martín-Moreno, F. J. García-Vidal, and A. Boltasseva, "Efficient unidirectional ridge excitation of surface plasmons," Opt. Express 17, 7228-7232 (2009).

[5] N. Fong, P. Berini, and R. N. Tait, "Characterization of Grating-Coupled Long Range Surface Plasmon Polariton Membrane Waveguides," Optics Express 23, 17421-17430 (2015).

[6] P. Berini, "Surface Plasmon Photodetectors and their Applications," Lasers \& Photonics Reviews 8, 197-220 (2014).

[7] M. L. Brongersma, N. J. Halas, and P. Nordlander, "Plasmon-induced hot carrier science and technology," Nat. Nanotechnol. 10, 25-34 (2015).

[8] S. Siadat Mousavi, A. Stöhr and P. Berini, "Plasmonic photodetector with terahertz electrical bandwidth," Appl. Phys. Lett. 104, 143112 (2014).

[9] S. Siadat Mousavi, A., Stöhr, P. Berini, "Ultra-fast Surface Plasmon III-V Photodetectors Based on Nanomonopoles," J. Lightw. Technol. 34, 4682-4687 (2016).

[10] M. W. Knight, H. Sobhani, P. Nordlander, and N. J. Halas, "Photodetection with active optical antennas," Science 332, 702-704 (2011).

[11]I. Goykhman, B. Desiatov, J. Khurgin, J. Shappir, and U. Levy, "Waveguide based compact silicon Schottky photodetector with enhanced responsivity in the telecom spectral band," Opt. Express 20, 28594-28602 (2012).

[12]N. Othman, P, Berini, "Nanoscale Schottky contact surface plasmon point detectors for optical beam scanning applications," Appl. Opt. 56, 3329-3334 (2017).

[13] M. Alavirad, A. Olivieri, L. Roy, P. Berini, "High-responsivity sub-bandgap hot-hole plasmonic Schottky detectors," Optics Express 24, 22544-22554 (2016).

[14] M. Alavirad, A. Olivieri, L. Roy, P. Berini, "Fabrication of electrically contacted plasmonic Schottky nanoantennas on silicon," Chinese Optics Letters 16, 050007 (2018)

[15] J. A. Dionne, K. Diest, L. A. Sweatlock and H. A. Atwater, "A metal-oxide-Si field effect plasmonic modulator," Nano Lett. 9, 897-902 (2009).

[16] S. Hassan, E. Lisicka-Skrzek, A. Olivieri, R. N. Tait, P. Berini, "Fabrication of a plasmonic modulator incorporating an overlaid grating coupler," Nanotechnology 25, 495202 (2014).

[17]A. Olivieri, C. Chen, S. Hassan, E. Lisicka-Skrzek, R. N. Tait, P. Berini, "Plasmonic Nanostructured Metal-Oxide-Semiconductor Reflection Modulators," Nano Lett. 15, 2304-2311 (2015).

[18] P. Berini, R. Charbonneau, S. Jetté-Charbonneau, N. Lahoud, G. Mattiussi, "Long-range surface plasmon-polariton waveguides and devices in lithium niobate," J. Appl. Phys. 101, 113114 (2007).

[19] R. El-Ganainy, K. G Makris, M. Khajavikhan, Z. H. Musslimani, S. Rotter, D. N. Christodoulides, "Non-Hermitian physics and PT symmetry, "Nature Photonics 14, 11 (2017)

[20]E. Karami Keshmarzi, R. N. Tait, and P. Berini, "Single-mode surface plasmon distributed feedback lasers," Nanoscale 10, 5914 (2018).

[21] C. Hahn, E. Karami Keshmarzi, S. H. Song, C. H. Oh, N. Tait, P. Berini, "Unidirectional Bragg Gratings Using Parity-Time Symmetry Breaking in Plasmonic Systems," IEEE J. Sel. Top. Quant. Electr. 22, 4600712 (2016)

[22] C. Hahn, Y. Choi, J. W. Yoon, S. H. Song, C. H. Oh, P. Berini, "Observation of exceptional points in reconfigurable non-Hermitian vector-field holographic lattices," Nature Communications 7, 12201 (2016)

[23] Y. Choi, C. Hahn, J. W. Yoon, S. H. Song and P. Berini, "Extremely broadband, on-chip optical nonreciprocity enabled by mimicking nonlinear anti-adiabatic quantum jumps near exceptional points," Nature Communications 8 , 14154 (2017).

[24] J. W. Yoon, Y. Choi, C. Hahn, G. Kim, S. H. Song, K.-Y. Yang, J. Y. Lee, Y. Kim, C. S. Lee, J. K. Shin, H.-S. Lee, P. Berini, "Time-asymmetric loop around an exceptional point over the full optical communications band," Nature $562,7725(2018)$ 